\title{
Tratamento ortodôntico preventivo na perda precoce de dentes decíduos: relato de caso clínico
}

\author{
Preventive orthodontic treatment in early loss of primary teeth: clinical case report
}

Tratamiento de ortodoncia preventivo en la pérdida temprana de dientes temporales: reporte de un caso clínico

\section{Resumo}

Objetivo: Esse estudo tem como objetivo apresentar o tratamento ortodôntico preventivo de uma criança na dentadura mista com perda precoce de dentes decíduos através do uso de mantenedores de espaço. Metodologia: Partindo da permissão da paciente por meio do Termo de Consentimento Livre e Esclarecido (TCLE) em partilhar sua imagem para devido fim, o presente estudo trata de um relato de caso clínico de cunho analítico descritivo (Pereira et al., 2018). Relato de Caso Clinico: Paciente de 7 anos e 5 meses, gênero masculino, pardo, compareceu à clínica de Atenção Integral Infantil acompanhado pela mãe, com queixa de dor no elemento 55. Apresentava higiene bucal deficiente e múltiplas lesões cariosas, tendo como plano de tratamento medidas de promoção de saúde bucal, restaurações e exodontias, seguida da instalação de mantenedores de espaço, em decorrência da exodontia precoce, para evitar futuros problemas oclusais, visto que já possui sua eficácia comprovada. Considerações Finais: Dessa forma, o uso de mantenedores de espaço se faz extremamente necessário.

Palavras-chave: Ortodontia preventiva; Mantenedor de espaço em ortodontia; Odontopediatria.

\begin{abstract}
Objective: This study aims to present the preventive orthodontic treatment of a child with mixed dentition with early loss of primary teeth through the use of space maintainers. Methodology: Based on the patient's permission through the Informed Consent Form (FICF) to share her image for the proper purpose, this study is a clinical case report of a descriptive analytical nature (Pereira et al., 2018). Clinical Case Report: A 7 years and 5 months old male patient, brown, attended the Comprehensive Child Care clinic accompanied by his mother, complaining of pain in element 55 . He presented poor oral hygiene and multiple carious lesions, with the plan of treatment measures to promote oral health, restorations and extractions, followed by the installation of space maintainers, due to early extraction, to avoid future occlusal problems, as its effectiveness has already been proven. Final Considerations: Thus, the use of space maintainers is extremely necessary.
\end{abstract}

Keywords: Preventive orthodontics; Space maintainer in orthodontics; Pediatric dentistry. 


\begin{abstract}
Resumen
Objetivo: Este estudio tiene como objetivo presentar el tratamiento de ortodoncia preventivo de un niño con dentición mixta con pérdida temprana de dientes temporales mediante el uso de mantenedores de espacio. Metodología: Con base en el permiso de la paciente a través del Término de Consentimiento Informado (FICF) para compartir su imagen con el propósito adecuado, este estudio es un reporte de caso clínico de naturaleza analítica descriptiva (Pereira et al., 2018). Reporte de Caso Clínico: Paciente varón de 7 años y 5 meses, moreno, acudió a la clínica de Atención Integral Infantil acompañado de su madre, quejándose de dolor en el elemento 55. Presentaba mala higiene bucal y múltiples lesiones cariosas, con el plan de medidas de tratamiento. para promover la salud bucal, restauraciones y extracciones, seguido de la instalación de mantenedores de espacio, como resultado de una extracción temprana, para evitar futuros problemas oclusales, ya que su efectividad ya ha sido probada. Consideraciones finales: Por lo tanto, el uso de mantenedores de espacio es extremadamente necesario.
\end{abstract}

Palabras clave: Ortodoncia preventiva; Mantenedor de espacio en ortodoncia; Odontología pediátrica.

\title{
1. Introdução
}

O desenvolvimento e o correto posicionamento da dentição decídua estão diretamente ligados ao crescimento e desenvolvimento da mandíbula e maxila e dos músculos faciais, além disso também tem relação com a respiração e com os hábitos bucais da criança (Lira et al., 2019), é responsável por uma boa fonação, pela função mastigatória e por uma oclusão adequada, pois são os dentes decíduos que guiam a correta irrupção dos dentes permanentes (Reddy et al., 2018).

A perda precoce é considerada quando a perda do elemento decíduo ocorre antes do tempo natural de esfoliação, isso acontece por diversos fatores como traumas durante uma queda ou até mesmo lesões de cárie extensas (Armenio e et al., 2018).

A cárie dentária é considerada uma doença crônica e muito comum na infância (Dias et al., 2018) e, de acordo com os dados do SB Brasil, as crianças mais acometidas por essa doença são de 5 a 12 anos, tendo maior incidência nas regiões Norte, Nordeste e Centro-Oeste (Brasil, 2012). Esta doença pode causar dor no paciente, comprometer a estética do sorriso e até mesmo uma futura má-oclusão, decorrente da perda precoce quando a cárie não é tratada (Araújo et al., 2018).

Para que esta perda precoce não gere má-oclusão, é importante que se faça a manutenção adequada do espaço deixado pelo elemento que foi perdido, com mantenedores de espaço que são eficazes para a preservação do espaço, ajudando na direção dos dentes permanentes que irão erupcionar futuramente (Silva et al., 2019) e evitando a diminuição do perímetro do arco dentário, pois pode gerar problemas relacionados ao alinhamento dos dentes (Nadelman et al., 2020).

Estes aparelhos podem ser divididos em fixos ou removíveis e funcionais ou não funcionais. Os mantenedores não funcionais são utilizados para que os dentes posteriores não mesializem e os anteriores não lingualizem, já os funcionais são utilizados quando o espaço é perdido mesio-distalmente e verticalmente. Os mantenedores removíveis são utilizados em casos que ocorre a perda de muitos dentes decíduos e são feitos com resina acrílica, dentes de estoque e são fixados através de grampos. Já os mantenedores fixos podem ser utilizados tanto quando a perda é unilateral quanto bilateral, são confeccionados com fios ortodônticos, fixados em uma banda ortodôntica que é cimentada no dente decíduo (Silva e et al., 2020).

Dentre os mantenedores fixos no arco inferior, o arco lingual de Nance é um mantenedor de espaço fixo e não funcional, indicado para perdas bilaterais, possui diversas vantagens como a fácil confecção, baixo custo, não precisa da colaboração do paciente, fácil higienização. Este aparelho é utilizado para que os elementos posteriores não se movimentem no sentido mesial e os anteriores no sentido lingual, porém, mesmo com o seu uso, o elemento antagonista pode extruir. Não impede que o sucessor permanente irrompa na cavidade oral e nem prejudica o desenvolvimento dos maxilares (Silva et al., 2016).

Como opção para evitar a movimentação, a barra transpalatina também é um dispositivo fixo e não funcional, é utilizado na arcada superior e pode ser fixado nos primeiros ou segundos molares, possui fácil confecção e várias indicações de aplicações clínicas, além de uma grande variedade de ativação e de possível controle tridimensional. Por esses motivos, vem sendo muito utilizado na Ortodontia (Santos et al., 2019). 
Dessa forma, o objetivo do presente estudo é apresentar o tratamento ortodôntico preventivo de uma criança na dentadura mista com perda precoce de dentes decíduos através do uso de mantenedores de espaço.

\section{Metodologia}

Partindo da permissão da paciente por meio do Termo de Consentimento Livre e Esclarecido (TCLE) em partilhar sua imagem para devido fim, o presente estudo trata de um relato de caso clínico de cunho analítico descritivo (Pereira et al., 2018), realizado na clínica odontológica do CEUNI Fametro, na disciplina de estágio supervisionado em Clínica Integrada II, do curso de graduação do Centro Universitário Fametro.

\section{Resultados e Discussão}

Paciente de 7 anos e 5 meses, gênero masculino, pardo, compareceu à clínica de Atenção Integral Infantil acompanhado pela mãe, com queixa de dor no elemento 55. Durante a anamnese a mãe relatou que a criança apresentava episódios de dor durante alimentação sendo preciso o uso de analgésico. Foi verificado que o paciente apresentava bom estado de saúde geral, sem histórico de hábitos deletérios, apresentava uma dieta cariogênica, e realizava a escovação uma vez por dia, quanto aos tratamentos odontológicos já realizados: profilaxia e extração.

No exame extraoral foi verificado que o paciente estava com o lado direito da face edemaciado, com limitação de abertura bucal e febre. No exame clínico foi verificado que o paciente estava no $1^{\circ}$ período transitório da dentadura mista, apresentava ausência do elemento 75 e o elemento dentário 55 estava com extensa cavitação envolvendo a dentina na região de gengiva livre por vestibular, com presença de fístula (figura 1).

Figuras 1 - Fotos iniciais da arcada inferior e superior do paciente, respectivamente.

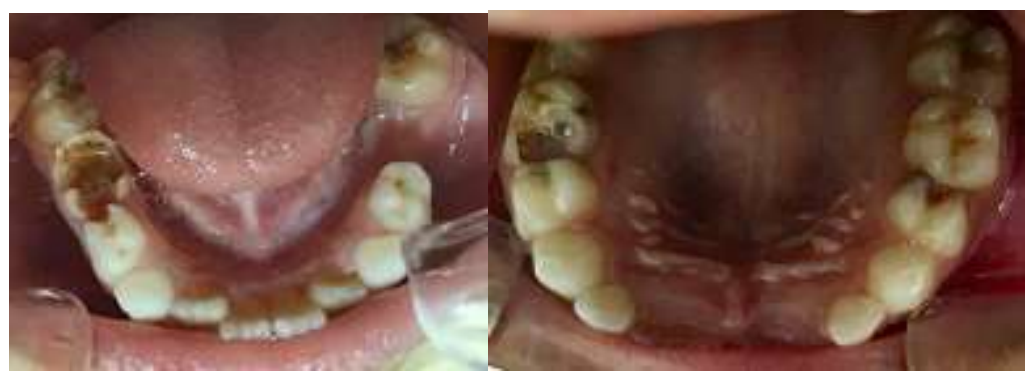

Fonte: Autores.

Foi então feita a prescrição de Amoxicilina suspensão oral durante 7 dias, e orientações quanto a higiene bucal para a responsável e para a criança. Após uma semana, o paciente retornou ainda com limitação bucal tornando-se inviável a realização do exame radiográfico e tratamento endodôntico.

Devido à urgência do procedimento optou-se pela exodontia do elemento 55. Posteriormente foi feita adequação do meio bucal e instalação de mantenedores de espaço, sendo realizada em sessões.

A primeira sessão consistiu na exodontia do elemento 85. Já na segunda sessão foi realizado selamento nos elementos 36 e 46, com ionômero de vidro restaurador Maxxion R - FGM e a prova da banda e moldagem da arcada superior para confecção da banda alça modificada. Na terceira sessão foi realizada restauração nos elementos 16 e 26, e instalação do mantenedor de espaço. Os elementos 73 e 74 foram restaurados na quarta sessão, em resina composta Aura Bulk Fill - SDI. Na quarta sessão o elemento 64 foi extraído. Na quinta sessão foi feita a prova das bandas inferiores e moldagem para confecção do arco lingual de Nance. A prova das bandas superiores e moldagem para confecção da barra transpalatina ocorreram na sexta sessão. Na sétima sessão foi realizada restauração em resina composta nos elementos 37 e 47. Na oitava sessão foi realizada 
restauração no elemento 65, também em resina composta Aura Bulk Fill - SDI. E, por fim, nas duas últimas sessões foi realizada a instalação dos mantenedores de espaço.

A cada consulta era realizada profilaxia e orientações sobre a escovação adequada e a sua importância para o paciente e para o responsável. Feita a adequação do meio, o tratamento de escolha para a perda precoce dos dentes decíduos foi a instalação de mantenedores de espaço, sendo eles: barra transpalatina no arco superior e arco lingual de Nance no arco dentário inferior, visto que, na radiografia panorâmica, foi verificado que os sucessores estavam no estágio 6 de Nolla (Figura 2).

Figura 2- Radiografia panorâmica do paciente.

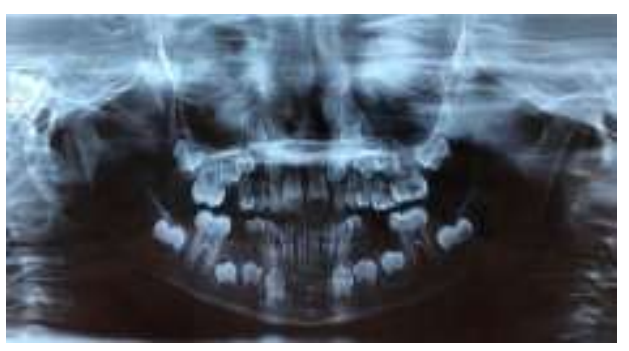

Fonte: Cimo.

Porém, de início, após a exodontia do elemento 55 foi instalado o mantenedor de espaço banda alça modificado, entretanto o paciente quebrou o aparelho com uma semana de uso e, devido à exodontia do elemento 64 posteriomente, optouse pela barra transpalatina (figura 3).

Figura 3 - Mantenedor de espaço banda alça modificado instalado no paciente;



Fonte: Autores.

Para a confecção dos dois aparelhos escolhidos, inicialmente foi feita a prova das bandas ortodônticas. Para a arcada superior a banda que melhor se adaptou aos molares 16 e 26 do paciente foi a U36 ${ }^{1 / 2}$ da marca Morelli. Para a arcada inferior a banda que se adaptou melhor foi a L37, também em ambos os molares. Em seguida foi feita a moldagem de transferência, de modo que as bandas ortodônticas foram adaptadas aos molares da criança e em seguida a moldeira perfurada infantil preenchida com alginato tipo II Jeltrate ${ }^{\circledR}$ Chromatic - Dentsply Sirona foi levada à boca do paciente, após a presa final do material de moldagem, as moldeiras foram retiradas e as bandas foram transferidas para o molde (Figura 4), que foi vazado com gesso pedra Durone IV - Dentsply Sirona. O modelo foi então enviado para o laboratório, para a confecção dos mantenedores de espaço (Figura 4). 
Figuras 4 - A) Prova das bandas ortodônticas superiores e inferiores, respectivaente; B) Moldagem das arcadas superior; C) Moldagem das arcadas inferios; D) Bandas ortodônticas adaptadas à moldagem superior; E) Bandas ortodônticas adaptadas à moldagem inferior; F) Vazamento do gesso na moldadem superior e inferior; G) Barra transpalatina e arco lingual de Nance confeccionados.
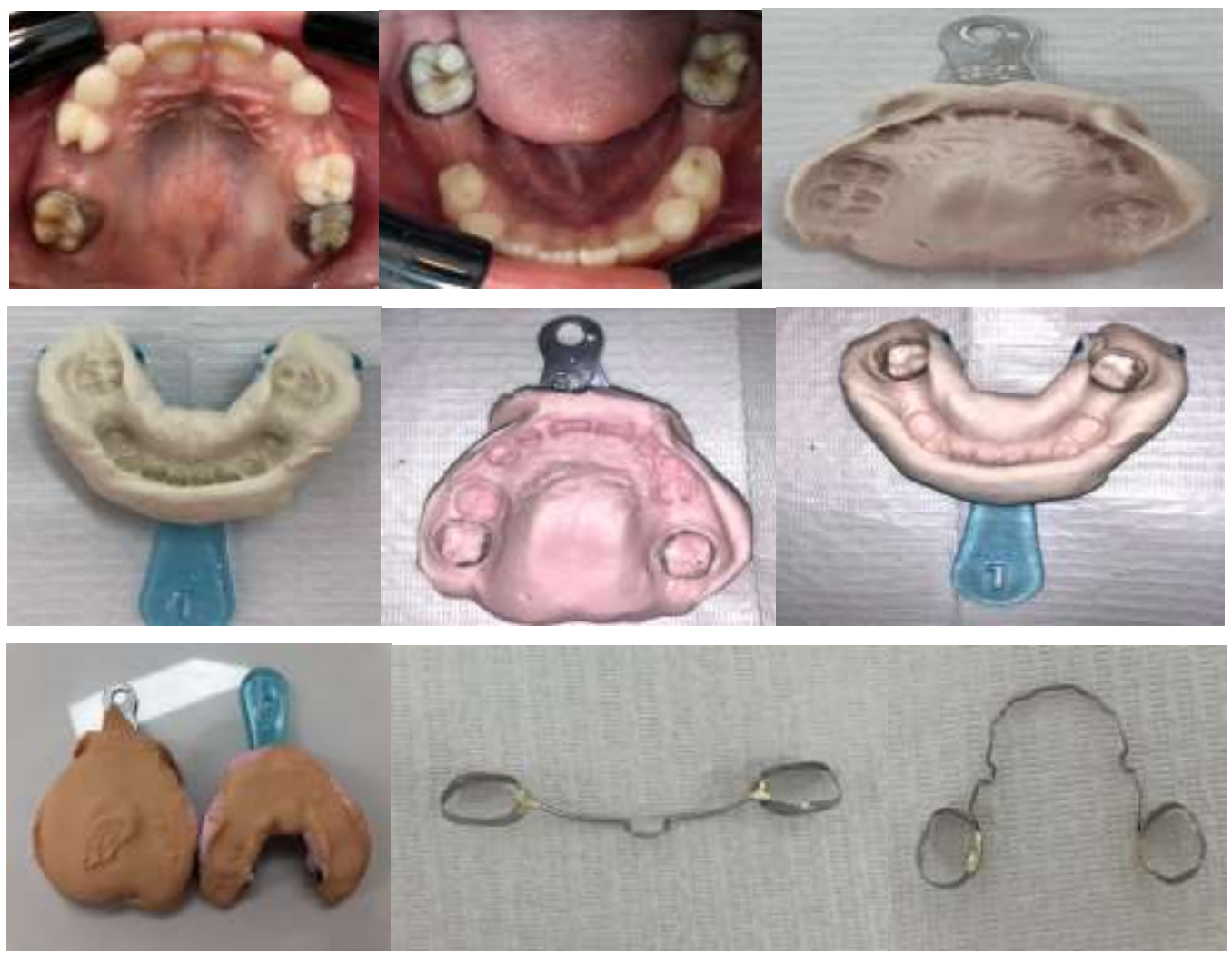

Fonte: Autoria própria.

Os mantenedores de espaço foram cimentados na boca do paciente com cimento de ionômero de vidro Ionglass C Maquira (Figura 5) e as orientações quanto aos cuidados e higiene foram passadas ao paciente e ao responsável.

Figuras 5 - Barra transpalatina e arco lingual de Nance instalados no paciente, respectivamente.

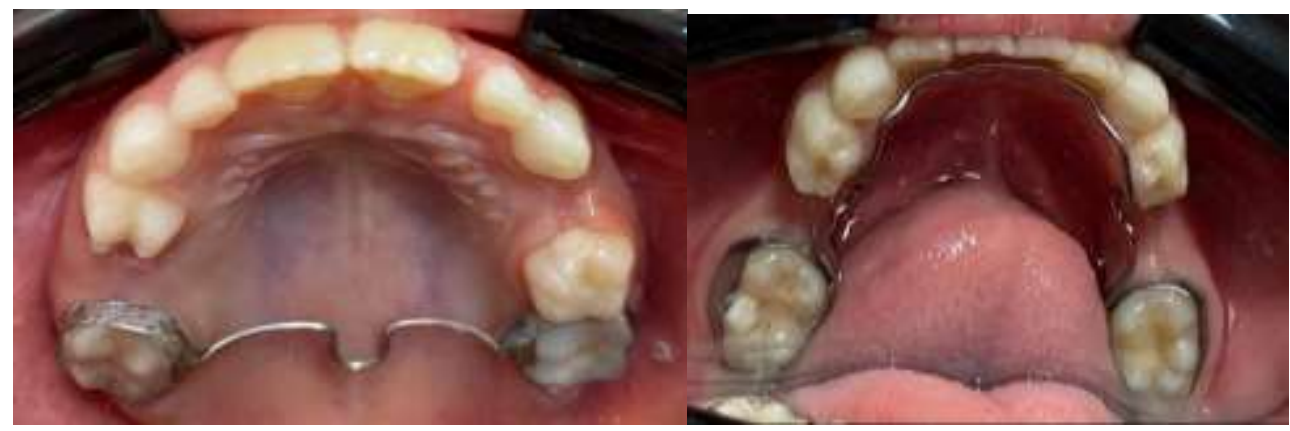

Fonte: Autoria própria.

Porém, devido à não colaboração do paciente e da família, a barra transpalatina se soltou 3 vezes (Figura 6), sendo necessária sua reinstalação e em todas elas as orientações eram reforçadas. Na última vez em que soltou, o paciente acabou perdendo o mantenedor de espaço, tornando-se necessária nova moldagem para confecção de um novo aparelho. Já o arco lingual de Nance precisou de apenas uma cimentação. 
Figura 6 - Barra transpalatina danificada após ser removida pelo paciente;

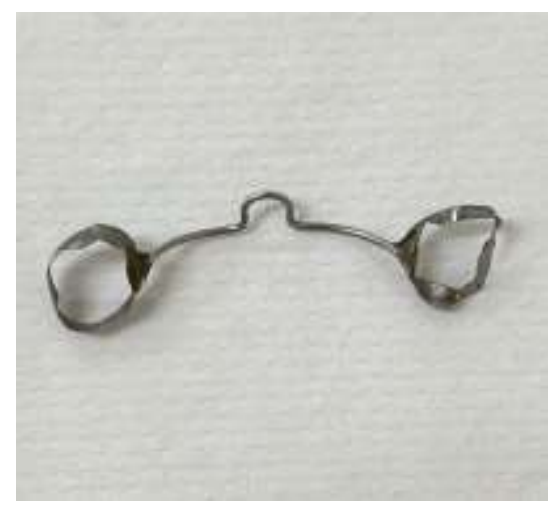

Fonte: Autoria própria.

O paciente ainda continua em acompanhamento, uma vez que, atualmente, possui 8 anos e os mantenedores de espaço precisam ser mantidos até que os pré-molares erupcionem, o que acontecerá por volta dos 10 aos 12 anos de idade.

\section{Discussão}

A perda precoce de dentes decíduos gera muitas consequências, principalmente quando ocorre em molares decíduos, pois causam uma diminuição da arcada dentária. Além disso, quando essa perda ocorre em consequência da doença cárie ou por trauma, pode gerar consequências a curto prazo que resultam em desequilíbrio da oclusão, direta ou indiretamente (Guimarães \& Oliveira, 2017).

No presente caso, o paciente alto risco de cárie dentária, presença de múltiplas lesões, tendo como plano de tratamento restaurações e exodontias e reforço da importância da higiene bucal tanto para a mãe quanto para criança, além de orientações quanto a importância da dieta para saúde bucal.

De acordo com Silva et al., 2020 torna-se imprescindível a adequada orientação ao paciente e aos responsáveis sobre a importância de manter o dente decíduo, uma vez que sua perda precoce pode gerar inúmeros problemas, como a movimentação dos demais elementos, dificultando a correta irrupção do sucessor permanente.

Porém, quando a perda precoce ocorre, a ortodontia preventiva se torna muito importante para que se faça a manutenção do espaço deixado, evitando a diminuição do comprimento do arco e outros problemas oclusais futuros (Silva et al., 2020). É responsabilidade do cirurgião-dentista as orientações ao paciente e responsáveis quanto à correta higienização e uso do dispositivo, evitando alimentos ricos em açúcar e alimentos pegajosos, para que o aparelho não se solte. Também deve ser evitado colocar a língua e os dedos no aparelho, para que não haja deformação do mesmo devido à pressão (Silva et al., 2016).

Devido as extrações precoces dos elementos decíduos bilateralmente tanto no arco inferior como no superior. Foram escolhidos dois mantenedores para a manutenção do espaço: o arco Lingual de Nance e a Barra Transpalatina.

O arco lingual de Nance possui um baixo custo e, além disso, não depende da colaboração do paciente. Ele é um arco passivo que possui suas extremidades soldadas ou encaixadas à bandas ortodônticas, localizado no terço cervical dos dentes inferiores e utilizado em casos em que ocorre a perda de forma bilateral. Este dispositivo impede que ocorra a mesialização dos molares e lingualização dos incisivos (Silva e et. Al., 2020) e não tem relação com o crescimento da mandíbula e da maxila. Deve ser instalado de forma que não interfira na irrupção dos elementos permanentes. Porém apresenta as desvantagens de não impedir a extrusão do dente antagonista e não devolver a função mastigatória. As bandas do arco podem ser cimentadas ao dente com ionômero de vidro, visto que possui boa adesão e boa resistência ao deslocamento, além também de liberar flúor (Silva et al., 2016). 
$\mathrm{Na}$ ortodontia preventiva a barra transpalatina é utilizada com a função de manter o perímetro da arcada dentária superior. Assim como o arco lingual, ela também possui suas extremidades soldadas às bandas ortodônticas e também possui as desvantagens de não reestabelecer a função mastigatória e não impedir a extrusão do elemento antagonista (Moreno e et. Al., 2018), porém apresenta como vantagens a sua fácil confecção, baixo custo e não necessita da colaboração do paciente para que tenha sucesso no tratamento, além disso, é um aparelho muito eficiente (Santos et al, 2019).

É importante que o paciente tenha um acompanhamento radiográfico, pois através da radiografia é possível verificar o estágio de desenvolvimento do germe do elemento sucessor permanente, sendo possível ter uma previsão de quando o mesmo irá irromper na cavidade oral, pois estudos apontam que o dente permante erupciona quando possui dois terços de sua raiz formados (Franco et al., 2021).

O paciente, bem como a família, não foi colaborativo. Diversas vezes a barra transpalatina foi retirada pelo mesmo, além disso não apresentou melhora na higiene oral. Os responsáveis sempre eram orientados e informados sobre a importância de uma higiene oral adequada e do uso dos mantenedores de espaço, para que não houvesse problemas oclusais futuramente.

A participação da família é indispensável, para incentivar a criança no tratamento e, além disso, através do vínculo criado entre a família e a equipe de saúde bucal, o paciente receberá um acompanhamento contínuo suprindo suas necessidades odontológicas (Figueiredo et al., 2020).

Esse estudo torna-se importante pois o uso de mantenedores de espaço em crianças que foram acometidas pela perda precoce de dentes decíduos é indispensável, visto que esses dispositivos auxiliam na prevenção do aparecimento de futuros problemas oclusais, como a incorreta irrupção dos elementos permanentes em consequência da movimentação dos dentes adjacentes, e já tem a sua eficácia comprovada.

\section{Conclusão}

A perda precoce de dentes decíduos, pode gerar inúmeros problemas oclusais, dentre eles a movimentação dos elementos adjacentes e a extrusão do antagonista, interferindo diretamente na correta erupção do sucessor permanente. Dessa forma, o uso de mantenedores de espaço se faz extremamente necessário.

\section{Referências}

Araújo, L. F. D., Alexandria, A. K., Letieri, A. D. S., \& Soares, T. R. C. (2018). Cárie precoce da infância: uma visão atual em odontopediatria. Rev. UNINGÁ, Maringá, 55, S3, 106-114.

Armenio, R., Costa, M. M. T. D. M., \& Garrastazu, M. D. (2018). Uso de mantenedor de espaço fixo não funcional em dentição decídua - relato de caso. Ação Odonto, (2). Santa Catarina.

Brasil. (2012). Ministério da Saúde. SB Brasil 2010: Pesquisa Nacional de Saúde Bucal: resultados principais. Brasília: Ministério da Saúde.

Dias, G. F., Ritzmann, B. F., Ransolin, F., \& Ferraz, T. R. K. (2018). Reabilitação estética e funcional em paciente com cárie precoce da infância: relato de caso. Rev. Odontol. Univ. Cid. São Paulo, 30(3), 314-22.

Figueiredo, R. C. D., Melo, R. H. V. D., Rodrigues, M. P., Souza, G. C. D. A., \& Vilar R. L. A. D. (2020). Experiência de atuação interprofissional do dentista na estratégia saúde da família. Revista Ciência Plural, 6(2), 21-43.

Franco, F. C. M., Araújo, T. M., \& Nascimento, A. C. S. (2021). Manutenção de espaço: da etiologia à interceptação. J Dent Public Health. 2021; 12(1):32-38.

Guimarães, C. D. A., \& Oliveira, R. C. G. (2017). Perda precoce de dentes decíduos: relato de caso. Revista UNINGÁ Review, Paraná, $29(2), 28-33$.

Lira, A. L. S., Costa, A. L., Fonseca, G. H. A., Silva, N. R. F., \& Martins, K. R. J. (2019). Deciduous tooth early loss prevalence in posterior region and indication of band-loop space maintainer. Brazilian Dental Science, 22(3).

Moreno, A. P. P., Aguiar, A. P. D., Junior, L. E. A., Crepaldi, M. D. L. S., Sant'ana, A. P., \& Crepaldi, A. A. (2018). Recuperação de espaços em dentadura mista com uso de aparelho hyrax, barra transpalatina e aparelho fixo 4x2. Revista FAIPE, 8(2), 8-20.

Nadelman, P., Bedran, N., Magno, M. B., Masterson, D., Castro, A. C. R., \& Maia, L. C. (2020). Premature loss of primary anterior teeth and its consequences to primary dental arch and speech pattern: A systematic review and meta-analysis. International Journal of Paediatric Dentistry. 30(6), 687-712. 
Research, Society and Development, v. 10, n. 16, e309101624028, 2021

(CC BY 4.0) | ISSN 2525-3409 | DOI: http://dx.doi.org/10.33448/rsd-v10i16.24028

Pereira, A. S., Shitsuka, D. M., Parreira, F. J., \& Shitsuka, R. (2018). Metodologia da pesquisa científica. Santa Maria, RS: UFSM, NTE.

Reddy, N. V., Daneswari, V., Shruti, G., Reddy, H., Reddy, A., \& Reddy, S. (2018). Premature loss of primary teeth on arch dimensions in 6- to 10-year-old schoolchildren in Khammam town, Telangana state. Int J Pedod Rehabil, 3, 67-71.

Santo,s M. P., Santos, D. C. L., Flaiban, E., Negrete, D., \& Santos, R. L. (2019). Barra transpalatina, características e aplicações clínicas: revisão de literatura. Rev.Odontol. Univ. Cid. São Paulo, (3) 48-60.

Silva P. V., Noberto, J, S, L., Del Papa, A. B. R., Simões, C. A. D., Berger, S. B., \& Aranha, A. M. F. (2019). Aesthetic-Functional Fixed Appliance as Treatment of Premature Loss of primary Anterior Teeth. J Health Sei, Mato Grosso.

Silva, A. A. D., Dantas, A. C. B., Araújo, A. L. D., Pessoa, M. E. H., Andrade, L. J., Ferrer, R. O., Gomes, C. E. B., Viana, J. R., Lucena, B. G., Germano, P. H. A., Goldfarb, E. C. A., \& Araújo, R. M. (2020). Exodontia do primeiro molar deciduo, seguido de adaptação de mantenedor de espaço tipo banda alça: Relato de caso. Braz. J. of Develop., Curitiba, 6(10), 80199 -80215.

Silva, L. A. M. D., Pinheiro, J. C., Silva, G. G. D., Monteiro, F. A. B. D. S., Porto, G. C. C., Silva, J. P. D. S., Rodrigues, D. D. A, Feitoza, V. A., Araujo, F. S., Moura, A. P. G. E., Santos, K. M. D. J., Silva T. F. D., Santos Junior, W. D., Andrade, A. S., \& Silva, A. F. D. (2020). Use of space maintainers and recoverers in interceptative orthodontics: Review of current concepts. Research, Society and Development, 9(11), e1009119627.

Silva, M. C., Barbosa, C. C. N., Barbos, O. L. C., \& Brum, S. C. (2016). Arco Lingual de Nance - sugestão de protocolo de instalação: relato de caso. Revista Pró-UniverSUS. 07 (3), 08-14. 